\title{
Bone Marrow-Derived Nonreactive Astrocytes in the Mouse Brain After Permanent Middle Cerebral Artery Occlusion
}

\author{
Zsuzsanna E. Tóth,, Ronen R. Leker, ${ }^{3}$ Tal Shahar, ${ }^{3}$ Andras Bratincsak, ${ }^{4}$ Ildiko Szalayova, \\ Sharon Key, ${ }^{1}$ Miklós Palkovits, ${ }^{2}$ Riccardo Cassiani-Ingoni, ${ }^{3}$ and Eva Mezey ${ }^{1}$
}

We studied the effect of permanent unilateral middle cerebral artery occlusion (PMCAO) on the generation of bone marrow (BM)-derived astrocytes in female mice previously transplanted with enchanced green fluorescent protein-expressing BM from male donors. In addition to an untreated PMCAO group, one group of mice also received intracerebral infusion of transforming growth factor-alpha, resulting in a decrease in the size of the infarct. Two months after PMCAO, we found a specific type of astrocyte of BM origin in the side of the injury, near the lesion. These astrocytes did not express glial fibrillary acidic protein (GFAP) by conventional fluorescence immunostaining; however, GFAP was easily detectable by tyramide signal amplification. These cells also expressed S100ß, confirming their astrocytic character. Unlike the endogenous reactive astrocytes, these BMderived astrocytes did not proliferate during the first week of ischemia and did not contribute to the glial scar formation. Transforming growth factor-alpha infusion increased the number of BM-derived astrocytes, without affecting their distribution. Interestingly, exclusively by tyramide signal amplification staining, we found that endogenous astrocytes displaying an identical morphology were also present in control mouse and human brains. Our data demonstrate that a subpopulation of nonreactive astrocytes expressing low levels of GFAP can originate from transplanted BM in the ischemic brain. We believe that these cells represent a subpopulation of astrocytes earlier considered to be GFAP negative. The high number of astrocytes with identical morphology and chemical character in control brains suggest that these type of astrocytes may have important functional role in the central nervous system that calls for further studies.

\section{Introduction}

A STROCYTES ARE A subtype of macroglial cells in the central nervous system (CNS). They provide the optimal environment for neuronal communication [1,2], affect synaptic transmission, respond to neurotransmitters, and send chemical signals to neurons [3]. Additionally, astrocytes are essential in controlling the formation and development of synapses [4]. Most types of astrocytes express glial fibrillary acidic protein (GFAP) to some degree; fibrous astrocytes in the white matter express large amounts of GFAP, whereas protoplasmic astrocytes in the gray matter express less [5].

In case of a CNS injury intense degeneration and regeneration processes are induced simultaneously. A variety of growth factors play an important role in these processes. Transforming growth factor-alpha (TGF $\alpha$ )-acting through the epidermal growth factor receptor-exerts a neuroprotective effect in permanent unilateral middle cerebral artery occlusion (PMCAO), as well as after transient focal ischemia in rats $[6,7]$. TGF $\alpha$ also increases the number and the survival of neural stem cells [8], and induces their differentiation toward neuronal and glial fates [9-11].

Several independent groups have shown therapeutic benefit of intravenous infusion of bone marrow (BM)derived progenitor cells after stroke [12-19]. It has also been recognized that these cells may serve as precursors for different types of brain cells, including mainly microglia, but also neurons and astrocytes [20-24]. It was reported previously that BM-derived, GFAP-positive astrocytes were preferentially present in the ischemic hemisphere $48 \mathrm{~h}$ after unilateral PMCAO, but the specific subtype of these astrocytes was

\footnotetext{
Part of the results has been presented as a poster on the IBRO 2010 meeting at Pécs, Hungary.

${ }^{1}$ National Institute of Dental and Craniofacial Research, National Institute of Health, Bethesda, Maryland.

${ }^{2}$ Neuromorphological and Neuroendocrine Research Laboratory, Department of Anatomy, Histology and Embryology, Semmelweis University and the Hungarian Academy of Sciences, Budapest, Hungary.

${ }^{3}$ National Institute of Neurological Disorders and Stroke, Bethesda, MD.

${ }^{4}$ National Institute of Mental Health, Bethesda, Maryland.
} 


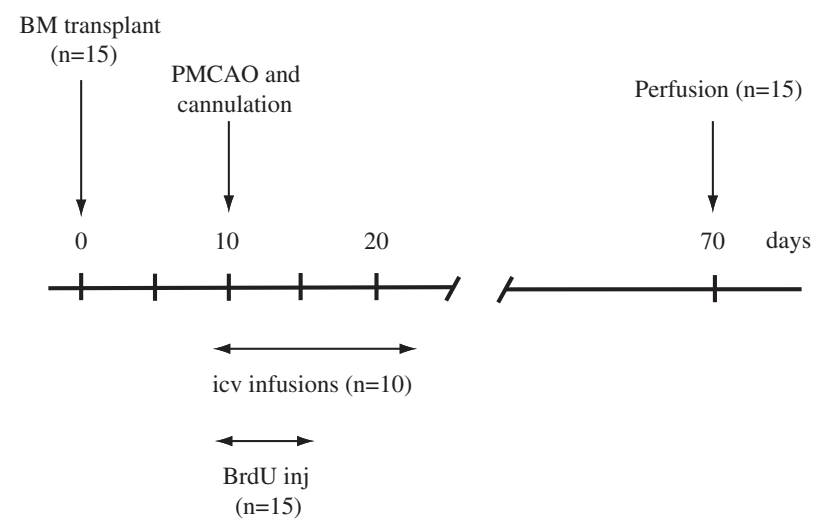

FIG. 1. Summary of the experimental design.

not determined [25]. In the present study, we investigated the distribution and the characteristics of BM-derived astrocytes following unilateral PMCAO after a survival period of 2 months. We also examined the potential effect of intracerebrally administered TGF $\alpha$ on the generation of astrocytes from transplanted $\mathrm{BM}$ precursors.

\section{Materials and Methods}

\section{Surgical procedures}

All experiments were approved by the institutional animal care and use committee and were conducted according to National Institutes of Health guidelines. Four- to six-weekold female C57B6 mice $(N=15)$ were subjected to irradiation [2×4.5 Gy (450 rad) $6 \mathrm{~h}$ apart] to deplete their own BM, and immediately after the second irradiation, they were given $\mathrm{BM}$ transplants generated from male mice that ubiquitously express (with the exception of erythrocytes) enhanced green fluorescent protein (GFP) [26] and kept in a sterile environment [23]. After 10 days of recovery, 10 mice were subjected to unilateral PMCAO the other 5 animals served as uninjured-BM transplanted controls. Shortly, the animals were anesthetized and placed in a tiltable stereotaxtic head holder (Kopf Instruments). Surgery was performed under a surgical microscope (Zeiss OPMI-1). The temporal muscle was removed and the temporo-zygomatic suture identified. The middle cerebral artery was exposed all the way down to its cross with the inferior cerebral vein by using a dental foot operated hand held drill and a bone cutter. The dura was reflected off the brain by a 23-gauge bevelled needle. Finally, the artery was occluded by electrocautery as it was carefully traced off the brain surface. At the completion of the surgery gel foam was placed over the exposed brain and the skin sutured [27]. This model results in cortical injury limited to a part of the frontal and parietal cortex while sparing subcortical structures. Immediately after induction of ischemia, a cannula was inserted into the brain adjacent to the infarct border using the following stereotaxic coordinates: $1.00 \mathrm{~mm}$ posterior to the bregma; $2.50 \mathrm{~mm}$ lateral to the midline; $3.00 \mathrm{~mm}$ ventral from the surface of the skull. Cannulas were attached to a sterile ALZET 1002 mini-osmotic pump delivering $0.25 \mu \mathrm{L} / \mathrm{h}$ (ALZET Osmotic Pumps Company) via sterile polyethylene tube and were fixed to the skull with sterile acrylic dental cement. The pumps contained either TGF $\alpha$ (20 ng/mL; Peprotech) or commercially available sterile artificial CSF (Harvard Apparatus) $(N=5)$ containing the following ion concentrations in $\mathrm{mM}$ : Na 150; K 3.0; Ca 1.4; $\mathrm{Mg}$ 0.8; P 1.0; Cl 155; then, they were active for 2 weeks, after which they were removed and the animals were allowed to survive. All mice were given the cell proliferation marker, bromodeoxyuridine (BrdU) $(50 \mathrm{mg} / \mathrm{kg}$ twice daily intraperitoneally; Roche Applied Sciences) to follow cell proliferation on days 1 through 5 after PMCAO. All mice were transcardially perfused under anaesthesia using $4 \%$ buffered paraformaldehyde ( $\mathrm{pH} 7.4) 2$ months after PMCAO. The experimental procedure is summarized in Fig. 1. Additional 3 mice were perfused to use as healthy controls.

\section{Immunohistochemistry}

Perfused-fixed mouse brains were cryoprotected by immersion in increasing concentration of sucrose and then frozen on dry ice, and 10- $\mu$ m-thick serial coronal sections were cut on a cryostat. The sections were mounted on positively charged microscope slides and stored at $-80^{\circ} \mathrm{C}$ until used. For immunohistochemistry the sections were brought to room temperature (RT), washed in $0.1 \mathrm{M} \mathrm{pH} \mathrm{7.4} \mathrm{phosphate-}$ buffered saline 3 times for $3 \mathrm{~min}$, and blocked with Universal Blocking Reagent (Biogenex) for $10 \mathrm{~min}$ at RT. Since the BrdU immunostaining required antigen retrieval, the sections were microwaved for $5 \mathrm{~min}$ in $10 \mathrm{mM}$ citric acid buffer $(\mathrm{pH}$ 6.1) and then let cool down to RT in the same buffer. The primary antibodies were applied according to Table 1, diluted in 1\% bovine serum albumin containing $0.25 \%$ Triton-X 100 . Incubation in the primary antibody was followed by blocking of endogenous peroxidase activity using $3 \% \mathrm{H}_{2} \mathrm{O}_{2}$ for $15 \mathrm{~min}$. The secondary antibodies were as follows: anti-rabbit horseradish peroxidase (HRP) polymer conjugate (Superpicture;

Table 1. List of Antibodies Used and Parameters of Immunostaining

\begin{tabular}{|c|c|c|c|c|c|c|}
\hline & Source & Host & Dilution & Pretreatment & Incubation & Detection \\
\hline GFP & Invitrogen & Rabbit & $1: 40,000$ & None & $1 \mathrm{~h}$ at $\mathrm{RT}$ & $\alpha-\mathrm{Rb}-\mathrm{HRP}$, FITC-Tyr \\
\hline GFP & Chemicon & Chicken & $1: 5,000$ & None & $1 \mathrm{~h}$ at $\mathrm{RT}$ & $\alpha$-Chk-Biotin, ABC, FITC-Tyr \\
\hline GFP & Abcam & Rabbit & $1: 10,000$ & None & $1 \mathrm{~h}$ at $\mathrm{RT}$ & $\alpha-\mathrm{Rb}-\mathrm{HRP}$, FITC-Tyr \\
\hline BrdU & Accurate & Rat & $1: 200$ & Microwave & $\mathrm{O} / \mathrm{N}$ at $4^{\circ} \mathrm{C}$ & $\alpha$-Rat-HRP, A350-Tyr \\
\hline GFAP & DAKO & Rabbit & $1: 500$ & None & $\mathrm{O} / \mathrm{N}$ at $4^{\circ} \mathrm{C}$ & $\alpha-\mathrm{Rb}-\mathrm{HRP}, \mathrm{A} 594-\mathrm{Tyr}$ \\
\hline GFAP & Sigma & mouse & $1: 1,000$ & None & $\mathrm{O} / \mathrm{N}$ at $4^{\circ} \mathrm{C}$ & Cy3-conjugated primary antibody \\
\hline$S 100 \beta$ & Abcam & Rabbit & $1: 1,000$ & None & $\mathrm{O} / \mathrm{N}$ at $4^{\circ} \mathrm{C}$ & $\alpha-\mathrm{Rb}-\mathrm{HRP}, \mathrm{A} 594-\mathrm{Tyr}$ \\
\hline Iba1 & WAKO & Rabbit & $1: 1,000$ & None & $1 \mathrm{~h}$ at $\mathrm{RT}$ & $\alpha-\mathrm{Rb}-\mathrm{HRP}, \mathrm{A} 594-\mathrm{Tyr}$ \\
\hline
\end{tabular}

$\mathrm{RT}$, room temperature; HRP, horseradish peroxidase. 
Zymed-Invitrogen) applied for $30 \mathrm{~min}$; biotinylated donkey anti-chicken IgY (Jackson Immuno-Research) at 1:1,000 for $1 \mathrm{~h}$, or HRP-conjugated anti-rat IgG (Jackson ImmunoResearch) at 1:500 overnight. All were followed by incubation in a tyramide-fluorochrome conjugate for $10 \mathrm{~min}$ at RT. The AlexaFluor-594 or FITC-conjugated tyramides were used in $1: 2,000$ and 1:20,000, respectively. In case of a double staining with a second tyramide amplification step, a second peroxidase blocking step was inserted after the first tyramide reaction by adding $0.5 \%$ sodium-azide to the $\mathrm{H}_{2} \mathrm{O}_{2}$ solution to block the HRP still present from the first staining. When antibodies were derived from the same host, we used a microwave treatment step to eliminate any nonspecific cross reaction [28] The immunostainings were evaluated either with a Leica DM RE-7 fluorescence microscope equipped with a Leica TCS SP2 AOBS confocal microscope or with a DMI6000 Leica inverted fluorescence microscope equipped with a Hamamatsu Orca camera, motorized stage, and Volocity 4.01 software (Improvision). Data were acquired by the confocal microscope with the multitrack mode with scanning in frame mode using the $364,405,488$, and $543 \mathrm{~nm}$ laser excitations corresponding to excitation wavelenghts of AlexaFluor350, DAPI, FITC, and AlexaFluor488, as well as Cy3 and AlexaFluor594, respectively. The channels were recorded with the following emission ranges: AlexaFluor $350 ; 450 \pm 30 \mathrm{~nm}$, FITC and AlexaFluor $488 ; 520 \pm 20 \mathrm{~nm}$, Cy3; $560 \pm 40 \mathrm{~nm}$, AlexaFluor594; 590 LP. When capturing by the conventional fluorescence microscope Z-stacks were collected at $0.5-\mu \mathrm{m}$ intervals, and iterative restoration was performed at a $95 \%$ confidence level.

\section{Y chromosome hybridization}

Y chromosome was colocalized with GFP as described previously [29]. Following the GFP immunostaining using the rabbit anti-GFP antibody (Molecular Probes-Invitrogen) and FITC-Tyramide (Table 1), the sections were immersed in $10 \mathrm{mM}$ citric acid ( $\mathrm{pH}$ 6.1) and microwaved in a kitchen microwave ( $700 \mathrm{~W}$; GE) for $2 \times 5 \mathrm{~min}$ at $50 \%$ power after the liquid started to boil. The evaporated water was replaced with distilled water between and after the microwaving sessions, and the sections were left in the solution to cool for $2 \mathrm{~h}$ at RT. Microwave treatment inactivates any HRP activity that is present in the tissue (ie, endogenous HRP and/or HRP incorporated in reagents used in previous steps) [28]. The $Y$ chromosomal hybridization was performed as previously described, using a 1.5-kb RNA probe (pY3531B) generated against a repeat sequence of the mouse $\mathrm{Y}$ chromosome that was labeled with digoxigenin using a kit (Roche Applied Sciences) [23].

After the hybridization step and several washes (for details, see http://intramural.nimh.nih.gov/lcmr/snge/), the digoxigenin was detected with an HRP-conjugated antidigoxigenin antibody (1:600; Roche Applied Sciences) and observed using the TSA-Plus CY3 System (1:600; Invitrogen).

\section{Infarct size determination}

Forebrains of TGF $\alpha$ - and artificial cerebrospinal fluid (ACSF)-treated mice $(N=4)$ were cut into $12-\mu \mathrm{m}$-thick coronal serial sections at $100 \mu \mathrm{m}$ intervals 60 days after PMCAO. The sections were stained with Toluidine Blue and photographed. Because at that time the infarct tissue has already liquefied, the volume of the infarct was determined by subtracting the area of the lesioned hemisphere from that of the normal hemisphere (also excluding the ventricles) on each sections $(N=6-8)$ multiplied by $100 \mu \mathrm{m}$ and summarized per animal using the NIH Image $1.37 \mathrm{v}$ program [11]. Infarct size $={ }_{n} \Sigma$ (area of the lesioned hemisphere - area of the normal hemisphere) $\times 100$. Statistical analysis was performed using unpaired $t$-test.

\section{Cell counting}

The number of BM-derived astrocyte-like GFP-positive cells was determined by counting the number of the cells/ section manually and analyzing 10-15 sections per animal $(N=4-5)$ from the level of the infarct. The total number of the cells/section was determined by counting the DAPIpositive cell nuclei from photographs using the NIH Image J $1.37 \mathrm{v}$ program.

\section{Human tissue sample}

Temporal cortex sample were surgically removed from a 40-year-old male patient with therapy-resistant temporal lobe epilepsy, by partial temporal lobectomy. The seizure focus was identified by multimodal studies, including video-electroencephalography (EEG) monitoring, magnetic resonance imaging, single photon emission computed tomography, and/or positron emission tomography. The patient had no gross temporal lobe damage based on postoperative histological analysis. A written informed consent for the study was obtained from the patient before surgery. The study was approved by the ethics committee at the Regional and Institutional Committee of Science and Research Ethics of Scientific Council of Health (TUKEB 5-1/1996, further extended in 2005) and performed in accordance with the Code of Ethics of the World Medical Assotiation (Declaration of Helsinki).

The middle temporal gyrus was cut into 1.5-2.0-mm-thick coronal blocks and fixed immediately after removal from the skull in $4 \%$ paraformaldehyde and stored at $4{ }^{\circ} \mathrm{C}$ until use. The sample was cryoprotected in $20 \%$ sucrose solution overnight, and $50-\mu \mathrm{m}$-thick floating sections were cut in a cryostat. The immunostainings were performed similarly to the mice brain sections using the anti-GFAP (DAKO; rabbit) primary antibody in 1:100 dilution for traditional immunohistochemistry observed by AlexaFluor488-conjugated antirabbit IgG (1:500; Invitrogen) and in a 1:1,000 dilution for amplification with FITC-Tyramide.

\section{Results}

\section{Identification and characterization of BM-derived astrocytes in post-PMCAO brains}

Without enhancing the GFP signal by immunostaining, mostly microglial (microglial marker Iba1and GFP double positive) cells of donor origin were detected after PMCAO around the infarct area (not shown). We previously established that GFP signal intensity was decreased during the differentiation process in a high percentage of donor-derived cells. Because of the GFP signal decrease, these cells looked GFP-negative when using traditional detection techniques, despite they expressed GFP at a low level. To avoid this, we 
routinely used a much more sensitive technique, tyramide signal amplification (TSA) amplification to detect GFP [29-31]. Using GFP amplification we were able to observe $40 \%$ more cells of donor origin than using traditional staining techniques [29]. Following GFP amplification, a finely arborized type of cell with bush-like morphology was detected in the brains of mice that were irradiated, BM transplanted, and underwent PMCAO, but not in uninjured-BM transplanted controls. These donor-derived cells were rare, and only present on the side of the injury-mostly, but not exclusively, at the level of the injury, outside of the infarct in the gray matter (Fig. 2A, B). In addition to the GFP staining in the cytoplasm, the $\mathrm{Y}$ chromosomes in the cell nuclei of these cells were also simultaneously detected (Fig. 2C) to confirm the donor origin. The cells expressed the astrocytic cell marker GFAP, but only after the immunostaining was amplified by TSA, suggesting that they make very low levels of GFAP. The cell bodies and the proximal processes (where the amount of GFAP was probably the highest) were prevalently immunopositive (Fig. 2D-F). To further confirm the astrocytic characteristics of these cells, we also used another known astrocytic cell marker, S100 $\beta$. Most of the cells examined were $S 100 \beta$ positive, but we sporadically found $S 100 \beta$ negative ones too $(\sim 1$ out of 5$)$, suggesting a possible transitional state during their differentiation (Fig. 2GI). Interestingly, among the reactive astrocytes participating in the formation of the glial scar and gathering in the penumbral area, we never found GFP-positive, BM-derived astrocytes (Fig. 3A, B). The reactive, strongly GFAP-positive astrocytic population, morphologically recognizable from the elongated shape of the cells and the thickened main cellular processes without fine arborization, consisted exclusively from in high number proliferating, host-derived (ie, both GFP and Y chromosome negative) cells as indicated by double immunostaining for GFAP and BrdU in these GFP/Y-negative cells (Fig. $3 \mathrm{C})$. On the other hand, the GFP-positive, BM-derived astrocytes were never BrdU positive, indicating that they had not divided shortly after injury (when the BrdU was administered). Following TSA amplification of GFAP staining, similar finely arborized cells were found (particularly in the gray matter) in the control side of the brains that were weakly GFAP positive, but GFP negative (Fig. 3D).

\section{Observation of GFAP immunpositivity in astrocytes in the gray matter of control mouse and human brains}

We investigated control, nontransplanted brains to reveal whether there is a weakly GFAP-positive astrocytic population in the cortex. Using conventional immunostaining, only stellate astroglial cells were seen in the white matter (Fig. 4A). TSA amplification, however, uncovered a much greater number of astrocytes in the gray matter, with similar morphology to the GFP-positive, BM-derived astrocytes that we found 2 months after PMCAO. The morphological characteristics of these cells were the spherical shape, the thin main cellular processes, and the very extensive fine arborization (Fig. 4B, C). Localization of the astrocytes in the control cortices was topographically organized; they were present in the upper cortical layers and also showed $S 100 \beta$ positivity (Fig. 4D). They were detected not only in the cortex but also in the striatum and in the brainstem (not shown). When we determined that there is a significantly larger number of the low GFAP expressing, very finely arborized astrocytes in the mouse cortex in control animals, we wanted to see if this finding might be relevant to humans. Using cortical sections of human brains we repeated the GFAP staining and found that, indeed, the same population is present in the human brain and that signal amplification results in the observation of a large number of these types of astrocytes (Fig. 4E-H).

\section{Effect of TGF $\alpha$ on infarct size and the occurrence of GFP originated astrocytes}

TGF $\alpha$ administered into the brain near the infarct area caused a reduction in the size of the infarcts (ACSF: $21.4 \pm$ $3.6 \mathrm{~mm}^{3}$; TGF $\alpha: 8.7 \pm 3.5 \mathrm{~mm}^{3} ; P<0.05, N=4$ animal/group analyzing 6-8 sections per animal) and a 5 times increase in the percentage of the GFP-positive finely arborized cells (it elevated from $0.0024 \%$ to $0.012 \%$.of the total cell number) adjacent to the level of the infarct in the side of the injury, in the striatum (Fig. 2A). However, TGF $\alpha$ did not influence the appearance and distribution of the endogenous (nondonor BM originated) bush-like astrocytes or the distribution of the GFP-positive (ie, donor-derived) ones.

\section{Discussion}

Astrocytes represent a diverse population of cells [32]. Classification of astrocytes used to be based on mere morphology until specific markers such as GFAP and S100 $\beta$ became available. According to these protein markers, 2 main classes are distinguished: GFAP-positive and GFAPnegative astrocytes; the latter ones express GFAP mRNA but not the protein [33]. A recent review emphasizes that the morphology of the stained astrocytes is technique dependent [33]. Our results also point to the importance of the sensitivity of the detection system used, not only for the higher definition of morphological details, but because of the fact that the entire population of cells may remain invisible unless extremely sensitive techniques are used. When we used traditional fluorescent immunostaining, only a portion of the GFAP-positive astrocytes was detected in the cortex of nontransplanted control mice brains. However, after applying TSA amplification, we uncovered a large population of cells with very low GFAP expression; this is a population of cells that is at least equal in number to the cells detected by traditional staining. It probably represents the population of astrocytes earlier considered as GFAP negative, but GFAP mRNA positive [33]. Mature GFAP-negative endogenous astrocytes upregulate GFAP expression and proliferate to become reactive in case of injury [34]. This may explain the lack of mature GFAP-negative endogenous astrocytes in the cortex 2 months after PMCAO on the side of the injury at the level of the infarct in our experiments.

Interestingly, our results in fresh human surgical samples from cortex demonstrated that-similarly to mice-there is a significantly higher number of GFAP-positive astrocytes than it has been detected before. The human tissue sample originated from an epileptic patient during surgery (control human brain tissue sample can be obtained only postmortem, when degradation can occur), and seizures may evoke an upregulation of GFAP expression [35]. Despite this fact, we have shown the existence of a significant number of GFAP-expressing astrocytes detectable only with a very sen- 

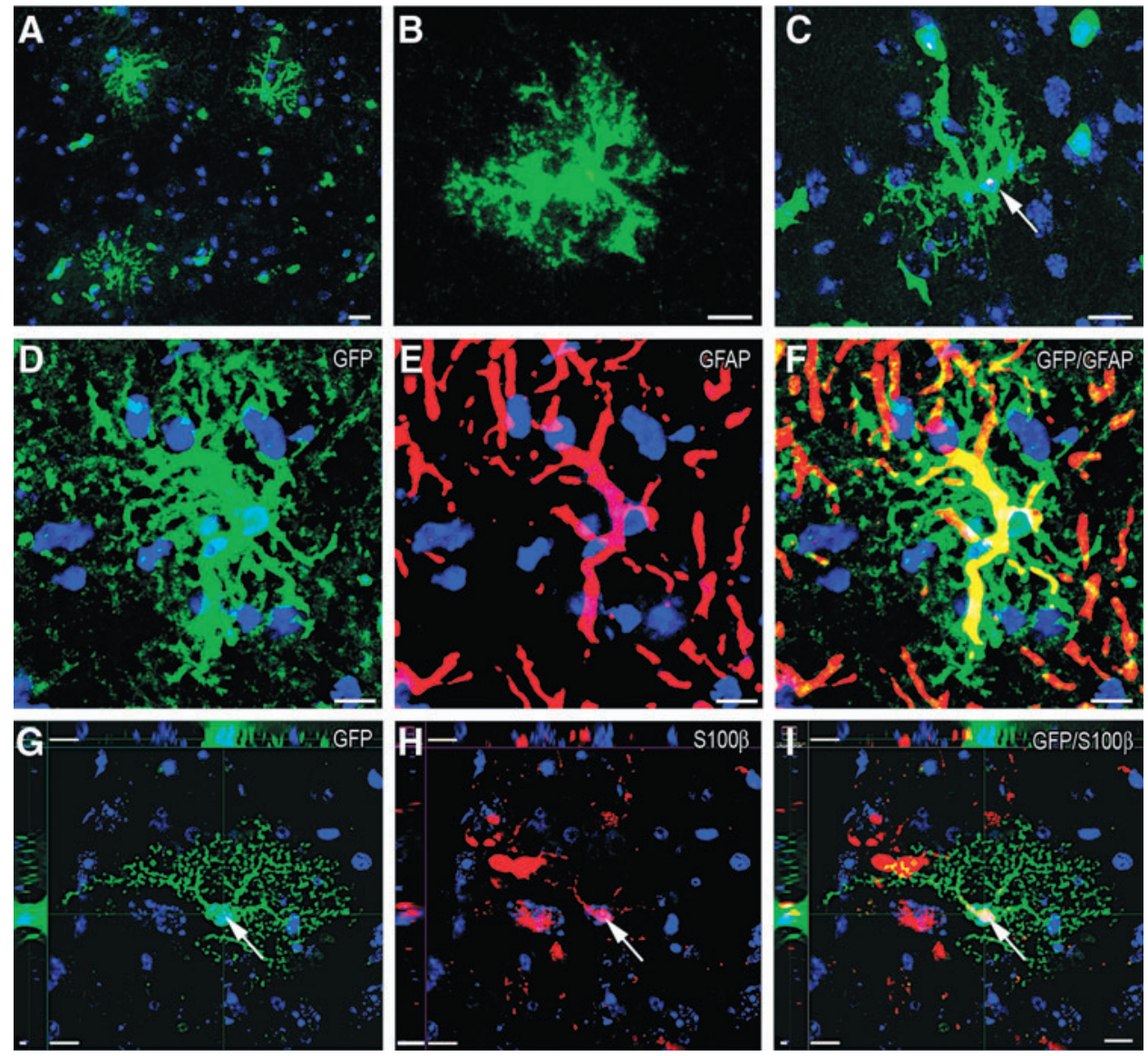

(red). The arrows point to the nucleus of the cell. (G) A GFP-positive (green) arborized cell. (H) S100 $\beta$ labeling (red) can be seen in the nucleus and in one of the processes of the same cell as in (G-I). An overlay of $\mathbf{G}$ and $\mathbf{H}$ confirms colocalization of GFP and S100 $\beta$ (yellow). Cell nuclei are counterstained with DAPI. Scale bars: (A-C); $20 \mu \mathrm{m},($ D-I); $10 \mu \mathrm{m}$. GFAP, glial fibrillary acidic protein; GFP, green fluorescent protein; PMCAO, permanent unilateral middle cerebral artery occlusion.


FIG. 3. Non-GFP labeled endogenous astrocytes after PMCAO. (A) The glial scar. Double immunohistochemistry for GFP (green), GFAP (red), and DAPI (blue). Only non-GFP-positive endogenous astrocytes participate in the formation of the scar. (B) GFP-negative, strongly GFAP-positive (red) reactive astrocytes in the penumbral area. (C) Many of the nonbone marrow-derived reactive astrocytes (GFAP, red) developed by proliferation (arrows), as seen by bromodeoxyuridine positivity (green). (D) Nonreactive astrocytes with weak GFAP expression in the control side of the cortex in the same section as on B, observed by TSA for GFAP (red). Cell nuclei are counterstained with DAPI. Pictures were taken at the level of the bregma $\pm 600 \mu \mathrm{m}$. Scale bars: A and D; $10 \mu \mathrm{m}, \mathbf{B}$ and C; $25 \mu \mathrm{m}$. TSA, tyramide signal amplification. 

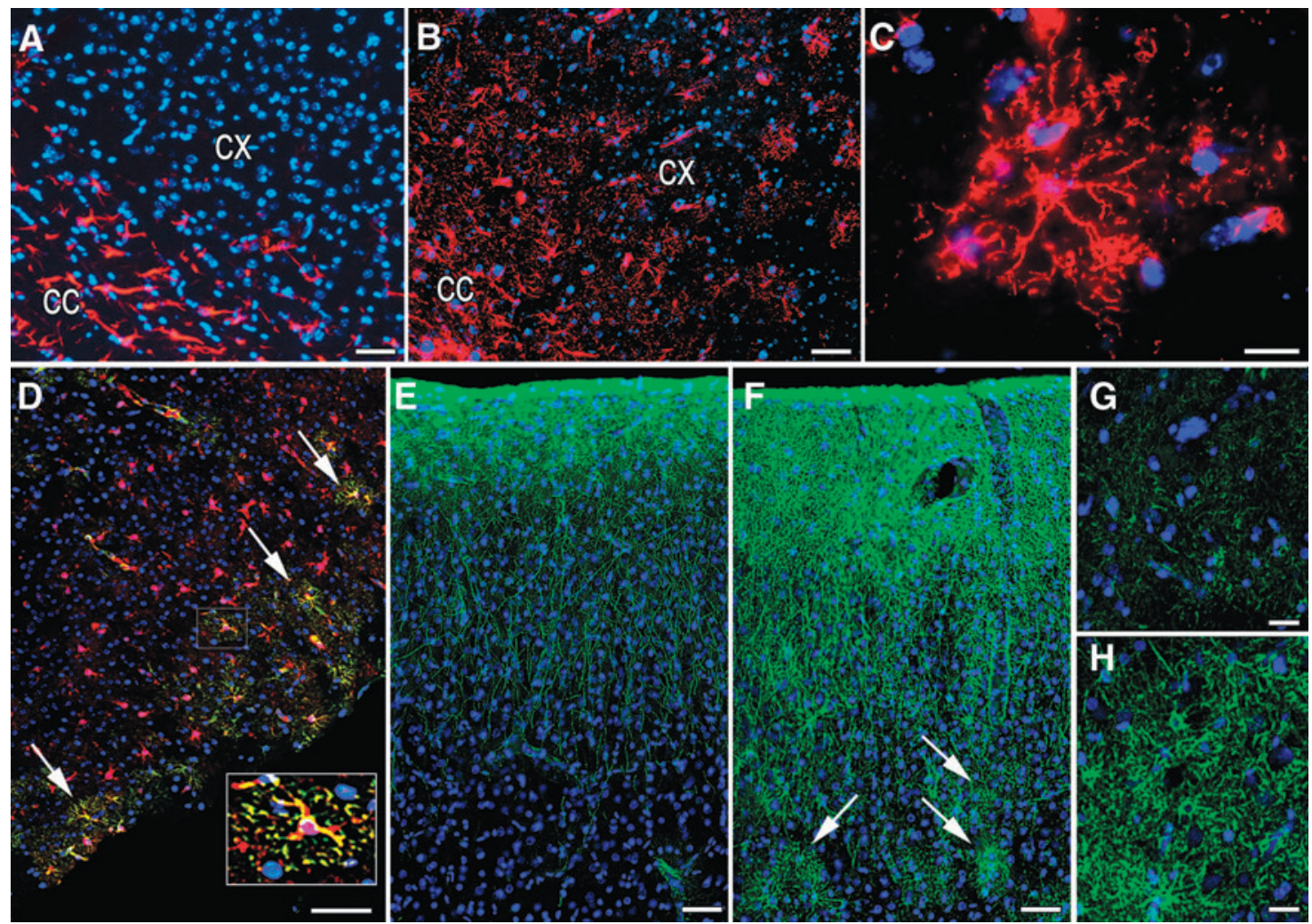

FIG. 4. Demonstration of astrocytes with weak GFAP positivity in healthy mouse and human brains. Importance of sensitivity of the methods. (A) Traditional immunostaining for GFAP (red) labels stellate astrocytes in the corpus callosum (cc). Note that the cerebral cortex (cx) is negative. (B) The same area as in A after GFAP immunostaining by TSA (red). A great number of additional, positively stained, finely arborized cells can be seen in the frontal cortex. (C) High power magnification picture of a cortical astrocyte, revealing the fine morphological structure of the branches. (D) GFAP labeled (green) astrocytes with delicate arborization express $\mathrm{S} 100 \beta$ (red) and are localized in the upper layers of the cortex (arrows). The inset shows a high magnification of an astrocyte (boxed in the image) with colocalizition of GFAP and (S100)ß. (E, F) Traditional and amplified immunostaining for GFAP in human temporal cortex, respectively. Similar type of astrocytes are seen as those detected in the mouse brain (B-D), which appear in a much higher number and with a more detailed morphology after the signal has been amplified (arrows). (G, H) Higher magnification images of cortical astrocytes in the human cortex immunostained by traditional method and by using TSA, respectively. Cell nuclei are counterstained with DAPI. Pictures of mouse brains were taken at the level of the bregma $\pm 600 \mu \mathrm{m}$. Scale bars: (A, B, E, F) $50 \mu \mathrm{m}$, (C) $20 \mu \mathrm{m}$, (D) $100 \mu \mathrm{m}(40 \mu \mathrm{m}$ for the inset), (G, H) $25 \mu \mathrm{m}$.

sitive method. This suggests a dramatical underestimation of the number of GFAP-expressing astrocytes in the human cortex, a relevant finding, since astrocytes seem to play an important role in the development of several neurological diseases, such as Alzheimer's disease and depression [36,37].

Using the amplification staining technique, we also identified BM-derived, weak GFAP-expressing astrocytes 2 months after PMCAO. These astrocytes were also GFAP negative by conventional immunofluorescence, and localized exclusively on the side of the infarct in the gray matter. The fact that they were not BrdU-positive suggests that they may have originated from circulating cells at a time-point different from the BrdU injections, or they differentiated into astrocytes after entering the ischemic brain without going through cell division. Theoretically, BM-derived cells might have also differentiated into BrdU-positive astrocytes that do not express GFP anymore, since about $10 \%$ of Y chromosome-positive cells downregulate GFP expression during differentiation [38]. However, in this case, we should have observed Y chromosome-positive, GFP-negative, weak GFAP-expressing cells; that did not happen. As it was mentioned, GFAP-negative astrocytes upregulate GFAP expression and proliferate to become reactive cells after injury [34,39]. Since, in our experiments, only endogenous astrocytes (ie, not GFP- and not Ypositive) participated in formation of the scar, the BM-derived astrocytes either represent a replacement population for the lost GFAP-negative astrocytes and/or may have an additionalso far unknown - role in the latter phase of CNS repair.

Several studies demonstrated the ability of circulating BM cells to enter the CNS and differentiate into GFAP-positive stellate astrocytes following PMCAO using conventional immunostaining. The percentage of GFAP-positive BM-derived cells varied in a wide range among the different studies, most likely depending on the type of injury, the route of administration, the concentration and preparation of BM cells, and the length of survival after PMCAO [20,40-42]. We did not find any stellate GFAP-positive BM-derived astrocytes after PMCAO in the white matter, but we found BM-derived nonreactive astrocytes in the gray matter by amplification of the GFAP signal. Chronic TGF $\alpha$ treatment 
(for 2 weeks starting at the time of the injury) reduced the infarct size, and increased the number of the specific type of BM-derived astrocytes that resembled cells of the mature astrocytic population of the healthy brain. TGF $\alpha$ is considered to play a prominent role in controlling astrogliosis [43], but was also shown to be neuroprotective (protecting against neuronal cell death and reducing infarct volume) when given before or after transient PMCAO [7]. Additionally, TGF $\alpha$ has recently been associated with almost complete behavioral recovery in a transient MCAO model [44].

The exact source of cells within the BM that differentiate into astrocytes is not known yet. Several studies have shown that BM stromal cells [also called mesenchymal stem cells (MSCs)] can differentiate into astrocytes in vitro $[45,46]$ and in vivo $[42,46,47]$. One group reported that intraventricularly grafted MSCs migrated in the brain, differentiated into astrocytes and that these cells were not a result of cell fusion [47]. In rats with PMCAO, up to $13 \%$ of the injected BMSCs became astrocytes, but-just as we observed-they did not participate in the formation of the glial scar [42]. A couple of studies suggested that even hematopoetic stem cells can differentiate into astrocytes if cultured in the astrocytic medium [48,49]. Given the above data, due to danger signals from the ischemic brain, one or more type of circulating BM-derived progenitors may home to the brain, migrate to the site of the injury, and take on astrocytic characteristics. Since the blood-born astrocytes have been shown to release growth factors, such as glial cell linederived neurotrophic factor (GDNF), nerve growth factor (NGF), and brain-derived neurotrophic factor (BDNF) [46], factors that can help injured neurons to survive they may help in decreasing the size and the functional consequences of the injury [50]. To address the exact role of these astrocytes in the CNS will need further investigations.

\section{Acknowledgments}

The research was supported by the Division of Intramural Research Program of NIDCR (E. Mezey), ETT 495/09 (Z.E. Toth), and by European Grant FP6 BNEII No. LSHM-CT2004-503039 (M. Palkovits). Z.E. Toth is supported by the Bolyai fellowship.

\section{Author Disclosure Statement}

None of the authors have any disclosure to declare. No competing financial interests exist.

\section{References}

1. Hansson E and L Ronnback. (2003). Glial neuronal signaling in the central nervous system. FASEB J 17:341-348.

2. Theodosis DT, DA Poulain and SH Oliet. (2008). Activitydependent structural and functional plasticity of astrocyteneuron interactions. Physiol Rev 88:983-1008.

3. Fellin T and G Carmignoto. (2004). Neurone-to-astrocyte signalling in the brain represents a distinct multifunctional unit. J Physiol 559:3-15.

4. Stevens B. (2008). Neuron-astrocyte signaling in the development and plasticity of neural circuits. Neurosignals 16:278-288.

5. Hajós F. (2008). A neuroglia szerkezete. In: Glia. Kalman M and Huszty LS, eds. Akadémiai Kiadó, Budapest, pp 18-28.

6. Justicia C and AM Planas. (1999). Transforming growth factor-alpha acting at the epidermal growth factor receptor reduces infarct volume after permanent middle cerebral artery occlusion in rats. J Cereb Blood Flow Metab 19:128132.

7. Justicia C, FJ Perez-Asensio, MC Burguete, JB Salom and AM Planas. (2001). Administration of transforming growth factor-alpha reduces infarct volume after transient focal cerebral ischemia in the rat. J Cereb Blood Flow Metab 21: 1097-1104.

8. Fallon J, S Reid, R Kinyamu, I Opole, R Opole, J Baratta, M Korc, TL Endo, A Duong, G Nguyen, M Karkehabadhi, D Twardzik, S Patel and S Loughlin. (2000). In vivo induction of massive proliferation, directed migration, and differentiation of neural cells in the adult mammalian brain. Proc Natl Acad Sci USA 97:14686-14691.

9. Cameron HA, TG Hazel and RD McKay. (1998). Regulation of neurogenesis by growth factors and neurotransmitters. J Neurobiol 36:287-306.

10. Junier MP. (2000). What role(s) for TGFalpha in the central nervous system? Prog Neurobiol 62:443-473.

11. Leker RR, ZE Toth, T Shahar, R Cassiani-Ingoni, I Szalayova, S Key, A Bratincsak and E Mezey. (2009). Transforming growth factor alpha induces angiogenesis and neurogenesis following stroke. Neuroscience 163:233-243.

12. Cairns K and SP Finklestein. (2003). Growth factors and stem cells as treatments for stroke recovery. Phys Med Rehabil Clin N Am 14:S135-S142.

13. Chen J, Y Li, L Wang, Z Zhang, D Lu, M Lu and M Chopp. (2001). Therapeutic benefit of intravenous administration of bone marrow stromal cells after cerebral ischemia in rats. Stroke 32:1005-1011.

14. Hess DC and CV Borlongan. (2008). Stem cells and neurological diseases. Cell Prolif 41 (Suppl 1):94-114.

15. Li Y, J Chen, CL Zhang, L Wang, D Lu, M Katakowski, Q Gao, LH Shen, J Zhang, M Lu and M Chopp. (2005). Gliosis and brain remodeling after treatment of stroke in rats with marrow stromal cells. Glia 49:407-417.

16. Li Y, M Chopp, J Chen, L Wang, SC Gautam, YX Xu and Z Zhang. (2000). Intrastriatal transplantation of bone marrow nonhematopoietic cells improves functional recovery after stroke in adult mice. J Cereb Blood Flow Metab 20:1311-1319.

17. Mezey E. (2007). Bone marrow-derived stem cells in neurological diseases: stones or masons? Regen Med 2:37-49.

18. Willing AE, J Lixian, M Milliken, S Poulos, T Zigova, S Song, C Hart, J Sanchez-Ramos and PR Sanberg. (2003). Intravenous versus intrastriatal cord blood administration in a rodent model of stroke. J Neurosci Res 73:296-307.

19. Zhang C, Y Li, J Chen, Q Gao, A Zacharek, A Kapke and M Chopp. (2006). Bone marrow stromal cells upregulate expression of bone morphogenetic proteins 2 and 4, gap junction protein connexin-43 and synaptophysin after stroke in rats. Neuroscience 141:687-695.

20. Eglitis MA and E Mezey. (1997). Hematopoietic cells differentiate into both microglia and macroglia in the brains of adult mice. Proc Natl Acad Sci USA 94:4080-4085.

21. Hickey WF and H Kimura. (1988). Perivascular microglial cells of the CNS are bone marrow-derived and present antigen in vivo. Science 239:290-292.

22. Mendez-Otero R, GR de Freitas, C Andre, ML de Mendonca, M Friedrich and J Oliveira-Filho. (2007). Potential roles of bone marrow stem cells in stroke therapy. Regen Med 2:417-423.

23. Mezey E, KJ Chandross, G Harta, RA Maki and SR McKercher. (2000). Turning blood into brain: cells bearing neuronal antigens generated in vivo from bone marrow. Science 290:1779-1782. 
24. Brazelton TR, FM Rossi, GI Keshet and HM Blau. (2000). From marrow to brain: expression of neuronal phenotypes in adult mice. Science 290:1775-1779.

25. Eglitis MA, D Dawson, KW Park and MM Mouradian. (1999). Targeting of marrow-derived astrocytes to the ischemic brain. Neuroreport 10:1289-1292.

26. Okabe M, M Ikawa, $K$ Kominami, $T$ Nakanishi and $Y$ Nishimune. (1997). "Green mice" as a source of ubiquitous green cells. FEBS Lett 407:313-319.

27. Leker RR, E Shohami, O Abramsky and H Ovadia. (1999). Dexanabinol; a novel neuroprotective drug in experimental focal cerebral ischemia. J Neurol Sci 162:114-119.

28. Toth ZE and E Mezey. (2007). Simultaneous visualization of multiple antigens with tyramide signal amplification using antibodies from the same species. J Histochem Cytochem 55:545-554.

29. Toth ZE, T Shahar, R Leker, I Szalayova, A Bratincsak, S Key, A Lonyai, K Nemeth and E Mezey. (2007). Sensitive detection of GFP utilizing tyramide signal amplification to overcome gene silencing. Exp Cell Res 313:1943-1950.

30. Bobrow MN, TD Harris, KJ Shaughnessy and GJ Litt. (1989). Catalyzed reporter deposition, a novel method of signal amplification. Application to immunoassays. J Immunol Methods 125:279-285.

31. Toda Y, K Kono, H Abiru, K Kokuryo, M Endo, H Yaegashi and M Fukumoto. (1999). Application of tyramide signal amplification system to immunohistochemistry: a potent method to localize antigens that are not detectable by ordinary method. Pathol Int 49:479-483.

32. Webster $\mathrm{H}$ and KE Astrom. (2009). Gliogenesis: historical perspectives, 1839-1985. Adv Anat Embryol Cell Biol 202:1-109.

33. Kimelberg HK. (2004). The problem of astrocyte identity. Neurochem Int 45:191-202.

34. Buffo A, I Rite, P Tripathi, A Lepier, D Colak, AP Horn, T Mori and M Gotz. (2008). Origin and progeny of reactive gliosis: a source of multipotent cells in the injured brain. Proc Natl Acad Sci USA 105:3581-3586.

35. Steward O, ER Torre, R Tomasulo and E Lothman. (1992). Seizures and the regulation of astroglial gene expression. Epilepsy Res Suppl 7:197-209.

36. Rajkowska G and JJ Miguel-Hidalgo. (2007). Gliogenesis and glial pathology in depression. CNS Neurol Disord Drug Targets 6:219-233.

37. Pelvig DP, H Pakkenberg, L Regeur, S Oster and B Pakkenberg. (2003). Neocortical glial cell numbers in Alzheimer's disease. A stereological study. Dement Geriatr Cogn Disord 16:212-219.

38. Toth ZE, RR Leker, T Shahar, S Pastorino, I Szalayova, B Asemenew, S Key, A Parmelee, B Mayer, K Nemeth, A Bratincsak and E Mezey. (2008). The combination of granulocyte colony-stimulating factor and stem cell factor significantly increases the number of bone marrow-derived endothelial cells in brains of mice following cerebral ischemia. Blood 111:5544-5552.

39. Fitch MT and J Silver. (1997). Glial cell extracellular matrix: boundaries for axon growth in development and regeneration. Cell Tissue Res 290:379-384.

40. Chen ZZ, XD Jiang, LL Zhang, JH Shang, MX Du, G Xu and RX Xu. (2008). Beneficial effect of autologous transplantation of bone marrow stromal cells and endothelial progenitor cells on cerebral ischemia in rabbits. Neurosci Lett 445 : 36-41.

41. Shen LH, Y Li, J Chen, Y Cui, C Zhang, A Kapke, M Lu, S Savant-Bhonsale and M Chopp. (2007). One-year follow-up after bone marrow stromal cell treatment in middle-aged female rats with stroke. Stroke 38:2150-2156.

42. Shen LH, Y Li, J Chen, A Zacharek, Q Gao, A Kapke, M Lu, K Raginski, P Vanguri, A Smith and M Chopp. (2007). Therapeutic benefit of bone marrow stromal cells administered 1 month after stroke. J Cereb Blood Flow Metab 27:6-13.

43. Rabchevsky AG, JM Weinitz, M Coulpier, C Fages, M Tinel and MP Junier. (1998). A role for transforming growth factor alpha as an inducer of astrogliosis. J Neurosci 18:1054110552.

44. Guerra-Crespo M, D Gleason, A Sistos, T Toosky, I Solaroglu, JH Zhang, PJ Bryant and JH Fallon. (2009). Transforming growth factor-alpha induces neurogenesis and behavioral improvement in a chronic stroke model. Neuroscience 160:470-483.

45. Fu L, L Zhu, Y Huang, TD Lee, SJ Forman and CC Shih. (2008). Derivation of neural stem cells from mesenchymal stem cells: evidence for a bipotential stem cell population. Stem Cells Dev 17:1109-1121.

46. Bahat-Stroomza M, Y Barhum, YS Levy, O Karpov, S Bulvik, E Melamed and D Offen. (2009). Induction of adult human bone marrow mesenchymal stromal cells into functional astrocyte-like cells: potential for restorative treatment in Parkinson's disease. J Mol Neurosci 39:199-210.

47. Deng J, BE Petersen, DA Steindler, ML Jorgensen and ED Laywell. (2006). Mesenchymal stem cells spontaneously express neural proteins in culture and are neurogenic after transplantation. Stem Cells 24:1054-1064.

48. Hao HN, J Zhao, RL Thomas, GC Parker and WD Lyman. (2003). Fetal human hematopoietic stem cells can differentiate sequentially into neural stem cells and then astrocytes in vitro. J Hematother Stem Cell Res 12:23-32.

49. Reali C, F Scintu, R Pillai, S Cabras, F Argiolu, MS Ristaldi, MA Sanna, M Badiali and V Sogos. (2006). Differentiation of human adult CD34+ stem cells into cells with a neural phenotype: role of astrocytes. Exp Neurol 197:399-406.

50. Park KW, MA Eglitis and MM Mouradian. (2001). Protection of nigral neurons by GDNF-engineered marrow cell transplantation. Neurosci Res 40:315-323.

Address correspondence to: Dr. Eva Mezey

National Institute of Dental and Craniofacial Research National Institute of Mental Health Bldg 49., Convent Drive Bethesda, MD 20892

E-mail: mezeye@mail.nih.gov

Received for publication April 16, 2010

Accepted after revision July 5, 2010

Prepublished on Liebert Instant Online July 6, 2010 